\title{
Early prediction of gait ability in patients with hip fracture
}

\author{
Eiki Tsushima $\cdot$ Ryukichi Hada $\cdot$ Manabu Iwata $\cdot$ \\ Hitoshi Tsushima
}

Received: 6 July 2008/ Accepted: 19 January 2009/Published online: 27 March 2009

(c) The Japanese Society for Hygiene 2009

\begin{abstract}
Objective Many elderly patients with hip fracture (HF) present with gait deficits. As such, an HF both indirectly and directly increases the number of elderly people requiring care, making it a major medical and economic problem in an aging society. To facilitate the treatment of $\mathrm{HF}$ and attempt to resolve the consequences, we have attempted to derive an equation that would predict gait ability. The prediction equation was developed by multivariate analysis using standard evaluation methods, with inclusion of guaranteed objectivity where possible. We attached greater importance to the prediction of gait ability early in the period of hospitalization, since this allows for early determination of an efficient therapeutic strategy.

Methods The subjects were $54 \mathrm{HF}$ patients (six men, 48 women; mean age: $78.0 \pm 8.4$ years) admitted to general hospitals in Hirosaki, Aomori prefecture, between 1998 and 2007. All were aged 60 years or older and were able to walk immediately before injury; physical therapy was initiated for all individuals during hospitalization. Evaluation items related to physical function, psychological function, and complications that may affect gait were evaluated; these included the manual muscle test, motor age test, Katz's index, dementia (HDS-R), consciousness disturbance, among others.
\end{abstract}

E. Tsushima $(\bowtie) \cdot$ M. Iwata $\cdot$ H. Tsushima

Graduate School of Health Sciences, Hirosaki University,

66-1 Hon-cho, Hirosaki, Aomori 036-8564, Japan

e-mail: pteiki@cc.hirosaki-u.ac.jp

R. Hada

Medical Informatics, University Hospital,

Hirosaki University, Hirosaki, Aomori, Japan
Results Based on data for 35 patients who could gait at discharge and 19 patients who could not, a model including MAT, HDS-R, and the New York Heart Association classification of cardiac function scores $(P<0.001)$ was obtained using multiple logistic regression analysis (discriminant hitting ratio: $94.4 \%$ ).

Conclusions The effectiveness of the derived model suggests that both physical and psychological functions should be considered for gait prediction.

Keywords Assessment of functional state . Gait on discharge · Hip fracture · Physical therapy · Prediction of gait ability

\section{Introduction}

Many elderly patients with hip fracture (HF) present with gait deficits. As such, an HF both indirectly and directly increases the number of elderly people requiring care, making this condition a major medical and economic problem in an aging society. Major factors that affect the prognosis of HF patients include dementia [1-3], delirium [4], extensor muscle strength of the knee joint [5], and complications $[6,7]$, such as cerebral stroke, heart disease, respiratory and circulatory disorders, and arthropathy. Improvements in surgical methods now enable the early initiation of rehabilitation, including physical therapy (PT). The probable duration of therapy and hospitalization can be estimated based on the above factors, and this allows the therapy to be performed efficiently. Choong et al. [8] reported that discussion of the treatment process and goals with the patient, the patient's family, and the medical staff led to shortening of the hospitalization period. In addition, early exercise with weight-bearing on the affected leg [9] 
and shortening of the hospitalization period [10] are effective approaches for functional recovery, as well as being of benefit financially.

While it has been accepted that the early prediction of the prognosis for physical function (gait ability) is likely to be of benefit for therapy, achieving such a prediction has two associated problems. The first is the difficulty in establishing concrete judgment criteria based on a cutoff point because the extent to which each of the above factors influences the prediction is unclear. Moreover, the degree to which each factor influences the prediction may vary depending on the specific combination of factors; consequently, only a rough prediction of gait ability can be obtained based on experience. The other problem is the lack of objective evaluation-based prediction criteria; information-sharing among physical therapists in this field would be greatly improved if gait ability could be predicted using an objective evaluation method with concrete indices of motor ability.

To resolve these problems, we have attempted to derive an equation for predicting gait ability, based on an investigation of the prognosis of gait at discharge, which is an important index of motor ability. The prediction equation was developed by multivariate analysis using standard evaluation methods, with the inclusion of guaranteed objectivity where possible. The time of evaluation was set to the day of initiation of weight-bearing exercise in HF patients; we attached greater importance to the prediction of gait ability early in the period of hospitalization, since this allows for early determination of an efficient therapeutic strategy.

\section{Methods}

\section{Participants}

The subjects were HF patients admitted to the general wards of district hospitals in Hirosaki, Aomori prefecture, between 1998 and 2007. The average duration of hospitalization for the HF patients was around 2 months. Patients aged 60 years or older who were able to gait immediately before injury and for whom PT was initiated during hospitalization were selected for the study. Patients who were admitted to hospital within 1 year, patients in whom PT was suspended or discontinued due to other diseases, and patients who died before discharge were excluded. Among those patients who satisfied these conditions, 54 patients (six men and 48 women) were randomly selected for inclusion in the study. Patients admitted to a single facility were selected to ensure uniformity in the procedures performed by the surgeon and the physical therapist in charge as well as consistency in the inpatient environment and the therapeutic policy of the staff. The items measured were essential to being able to evaluate and treat the functional states and were not harmful to the patients. The nature and purpose of these measurements were explained to the patients and their families, and consent was obtained before the evaluation was performed. Institutional ethics committee approval for the study was not obtained because such a committee had not yet been established at this time this study was initiated. All of the procedures were conducted in accordance with the Helsinki Declarations of 1975.

The mean age of the subjects was $78.0 \pm 8.4$ years (range 60-90 years), and the mean duration of hospitalization was $54.0 \pm 18.2$ days (range 24-134 days). All subjects underwent surgery: $\gamma$-locking nail, cannulated cancellous screw, multiple pinning, and captured hip screw were used for internal fixation in 36, two, one, and two patients, respectively. Thirteen patients underwent femoral head prosthetic replacement (Omnifit-UHR; Howmedica Osteonics, Allendale, NJ).

\section{Physical therapy procedure}

Physical therapy at the bedside was initiated 1 day after surgery and mainly involved range of motion exercise of the hip joint on the treated side and exercise for muscle strengthening. Weight-bearing gait training was initiated 3-5 days after surgery in patients who received osteosynthesis. and 14-16 days after surgery in patients who received femoral head prosthetic replacement, depending on the physical condition of the patient after surgery. Postponement of initiation of weight-bearing exercise due to insufficient fixation of the surgical region was not necessary in any of the study subjects. The physical therapist selected the appropriate assistive devices for training for standing and gait, such that the patients could utilize the exercise to their maximum ability, and appropriate changes to the assistive device were made when the gait ability of the patient improved. One of the authors (E. Tsushima) was the physical therapist in charge of PT for all subjects.

\section{Data collection}

With reference to several previous reports [1-7], items related to physical function, psychological function, and complications that may affect gait were evaluated (Table 1). Standard items that can be readily measured without a specific instrument were selected based on a consideration of general clinical applicability. Physical function was evaluated based on muscle strength, motor ability, and activities of daily living, and psychological function was evaluated based on intelligence and consciousness disturbance. 
Table 1 Evaluation criteria for each item

Physical function

Manual muscle test: The manual muscle test established by Daniels and Worthingham [19] evaluates muscle strength in six stages, ranging from 0 (no muscle contraction) to 5 (able to resist strong resistance). The hip joint flexor muscle strength and knee joint extensor muscle strength on the affected side were measured, and the average of the two measurements was used in the analysis

Motor age test (MAT): The MAT is based on an evaluation table in which typical motor items in normal childhood development are converted into scores. Scores are allocated to individual motor items. The score varies from 0 to 72 points (or 0-72 months), where 72 points represents the greatest motor ability

Katz's index (KI): Basic motions of daily living activity in a hospital room were evaluated at the time of initiation of exercise in a rehabilitation room. The KI evaluates whether the patient can perform the following six items by themselves or with assistance: bathing, dressing, going to the lavatory, movement, self-control of urination and defecation, and eating meals. The evaluation is made using seven categories, from A (all items are performed independently) to $\mathrm{G}$ (all items need assistance), based on the number of items performed independently

Psychological function

Hasegawa's dementia scale: This is an intelligence evaluation scale consisting of nine questions, and consistency with the Mini Mental State Examination is high. The evaluation is made based on the number of correct answers from 0 (all answers incorrect) to 30 (all answers correct)

Japan coma scale: Consciousness was judged as follows: 0 (clear consciousness), 1 (consciousness unclear), 2 (disorientation), 3 (the patient cannot give their own name and birth date), 10 (the patient opens their eyes in response to calling), and 20 (the patient opens their eyes in response to a loud voice or to shaking of his/her body)

Night delirium: Patients in whom night delirium was noted were judged $(+)$ and patients with no delirium were judged $(-)$. The judgment was made based on information obtained from nurses, with only a single episode of delirium required for a judgment of $(+)$ to be made

Complications

Influence of heart disease: Evaluation was made using the classification of cardiac function established by the New York heart association (NYHA). Patients with no heart disease and class-I and class-II heart diseases were judged as 'none', and patients with class-III and class-IV heart diseases were judged as 'mild' and 'severe', respectively

Influence of respiratory disorder: The Hugh-Jones classification was used. Patients with no respiratory disorder and grades I-IV respiratory disorders were judged as (-), and patients with grade $\mathrm{V}$ respiratory disorder were judged $(+)$

Influence of cerebral stroke: The legs were staged by Brunnstrom staging as I (severest paralysis) to VI (almost no paralysis)

Influence of other bone and joint disorders: Complications of rheumatoid arthritis, gonarthrosis, and compression fracture were classified into two categories, 'mild' and 'severe', corresponding to the degrees of functional disturbance and pain

For the evaluation of muscle strength, the hip joint flexor muscle strength and the knee joint extensor muscle strength on the affected side were evaluated based on the manual muscle test [11] (MMT), which is a simple and widely used test. For the evaluation of motor ability, leg motor function was evaluated by using the motor age test (MAT) reported by Johnson et al. [12] (Table 2). Activities of daily living were evaluated using the Katz's index (KI) [13]. When a test item could not be performed because the patient who reported pain refused to undergo the test or could not understand the content, the item was judged to be impossible to determine and was not included in the score.

For the evaluation of intelligence, the presence (in this case, also the severity) or absence of a decrease in intelligence was evaluated using the standard method in Japan, which is a revised version of Hasegawa's dementia scale (HDS-R) [14]. For the evaluation of the level of consciousness disturbance, the Japan coma scale (JCS) was used. When night delirium (henceforth referred to as delirium) was noted once or more during the period between admission and the evaluation day, a judgment of 'yes' was made regarding delirium.

For complications, the New York Heart Association classification of cardiac function [15] (NYHA) was used to evaluate heart disease, and the Hugh-Jones classification (HJ) [16] was used for respiratory disease. The Brunnstrom stage [17] of the legs was used for evaluating the degree of paralysis in patients with cerebral stroke. This evaluation method has a high reliability. Therefore, anyone can easily take the evaluation method. For patients with orthopedic disorders other than HF, subjective severity was evaluated using motor function as the index, because no corresponding standard evaluation method was available.

Physical and psychological functions were evaluated on the day weight-bearing exercise was initiated in a rehabilitation room; for example, standing up from a chair and gait training. Physical therapy was continued, and MAT and KI scores were re-evaluated 1 week after the initial evaluation. The MMT and HDS-R scores were not re-evaluated because no major changes were noted after 1 week. Age, the number of days after admission at the time of initiation of bedside PT, and the number of days after initiation of bedside PT that exercise in a rehabilitation room was started were also included in the analysis. A single examiner (E. Tsushima) performed all evaluations to increase examiner reliability.

The patients were divided into the walking group, which consisted of 35 patients who could gait at discharge, and 
Table 2 Motor age test (partial description)

\begin{tabular}{lll}
\hline Month & Item & Score \\
\hline 4 & Sits, back support, tall or long, on mat & 2 \\
7 & Head balance almost mastered & 2 \\
10 & Sits for 1 min on the floor, no support, tall or long & 3 \\
& Rolls, both ways, complete revolutions & 1 \\
& Stands holding on to furniture & 1 \\
12 & Crawls in any fashion, prone or in hands-and-knees position & 1 \\
& Crawls reciprocally, on hands-and-knees or on all fours & 1 \\
15 & Pulls up on furniture to standing position & 1 \\
& Walks with stop-start ability (four out of six attempts) & 3 \\
& Runs stiffly & 1 \\
21 & Moves up and down standard stairs with rail, any fashion & 1 \\
& Seats self in suitably sized chair with arms, away from wall & 1 \\
24 & Walks downstairs, holding tech. by one hand & 1.5 \\
& Walks upstairs, holding rail (one or two hands) & 1.5 \\
& Runs 50 feet without falling & 1.5 \\
\end{tabular}

the non-walking group, consisting of 19 patients who could not do so. When the patient could gait independently in the ward without attention, the patient was included in the walking group, regardless of whether an assistive device was required. This judgment was made based on the unanimous agreement of the physician, the physical therapist, and the nurses in charge of the patient.

\section{Data analysis}

We used multiple logistic regression (MLR) analysis [18] to prepare a prediction equation to differentiate between the walking and non-walking groups. As prior measures, bivariate statistical analysis was performed and significant items identified. For quantitative data, a two-sample $t$ test was applied. When the variance of the two samples was unequal in a Levene test, a Welch test was performed. For categorical data, a Mann-Whitney test and a $X^{2}$ test for independence (exact test when a category with an expected value of 5 or lower was present) were applied for items with many categories and few categories, respectively. Discriminant analysis using the stepwise method ( $p_{\text {in }}=0.25$ ) was performed for a selection of items, with classification of the walking and non-walking groups as dependent variables and all other items as independent variables.

Discriminant analysis is a parametric method, and it has limitations, including the assumption of a multivariate normal distribution and the necessity of continuity of data scales. Since most data did not show a normal distribution or the data scale was categorical, MLR analysis was selected because of its wide applicability.
The MLR analysis was performed using significant items (identified as described above) as independent variables and the walking (category number $=1$ ) and non-walking (category number $=0$ ) groups as dependent variables. To select the independent variables for MLR, we constructed an optimal model based on the likelihood ratiobased forward selection method satisfying the following conditions: (1) independent variables are lower than the significance level; (2) a -2 maximal likelihood (-2ML) reflects a significant decrease; (3) the increase in the model $X^{2}$ value is significant; (4) the discriminant hitting ratio is high. We noted that construction of a model based on these statistical values may lead to illogical results without ensuring consistency with medical common sense [18]. Thus, the investigation was repeated with inclusion of additional items that were likely to have an effect on prognosis based on medical and empirical knowledge. A linear relationship between the logit and changes in a given value was confirmed based on the $\log$ odds-ratio for the independent variables finally selected by MLR.

The $95 \%$ confidence interval $(95 \% \mathrm{CI})$ of the odds ratio (OR) of each item was calculated from the coefficient and standard error. To analyze the accuracy of the prediction equation, we calculated the predicted value using probability, and a cross table of the measured and predicted values was prepared based on values of $p>0.5$ for the walking group and $p<0.5$ for the non-walking groups, respectively, to obtain the discriminant hitting ratio. We used the SPSS ver.12.01 J software package (SPSS, Chicago, IL) for the above statistical analyses, and the significance level was set to $P=0.05$ or $P=0.01$. 


\section{Results}

The descriptive statistics for the evaluation items are shown in Table 3. Since all patients were of Brunnstrom stage III or IV (moderate paralysis), the classification was altered such that it was based on two categories: with (+) and without (-) paralysis. For orthopedic disorders other than HF, the patients had either no disorders or mild disorders, and thus were categorized as with (+) or without (-) orthopedic disorders.
The patients underwent periodic X-ray examination during hospitalization, and dislocation or deformity of the fractured region was confirmed. In one patient (an 89-year-old female in the walking group), the fractured region was dislocated, and the patient underwent gait training without weight-bearing for 2 weeks during hospitalization. In a number of other patients, PT was suspended for 2-3 days due to poor physical condition, but general therapy proceeded without major problems.

Table 3 Descriptive statistics for the items evaluated

\begin{tabular}{|c|c|c|c|c|c|c|c|c|c|c|}
\hline \multirow[t]{2}{*}{ Item $^{\mathrm{a}}$} & & & \multicolumn{3}{|c|}{ Walking group $(n=35)$} & \multicolumn{3}{|c|}{ Non-walking group $(n=19)$} & \multicolumn{2}{|c|}{ Statistics } \\
\hline & & & Mean \pm SD & Median & Range & \multirow{2}{*}{$\begin{array}{l}\text { Mean } \pm \text { SD } \\
82.7 \pm 5.4\end{array}$} & \multirow{2}{*}{$\begin{array}{l}\text { Median } \\
83.0\end{array}$} & \multirow{2}{*}{$\frac{\overline{\text { Range }}}{70-90}$} & \multirow{2}{*}{$\begin{array}{l}t \\
3.80\end{array}$} & \multirow{2}{*}{$\frac{P}{<0.001}$} \\
\hline Age (years) & & & $75.5 \pm 8.6$ & 75.0 & $60-90$ & & & & & \\
\hline MMT & & & $3.5 \pm 0.5$ & 4.0 & $3-4$ & $2.9 \pm 0.3$ & 3.0 & $2-3$ & 0.65 & $<0.001$ \\
\hline HDS-R (points) & & & $22.7 \pm 8$ & 24.0 & $6-30$ & $7.9 \pm 6.2$ & 7.0 & $0-21$ & 6.99 & $<0.001$ \\
\hline MAT (points) & & & $11.8 \pm 3.2$ & 12.0 & $7-22$ & $6.4 \pm 2.7$ & 7.0 & $2-12$ & 5.63 & $<0.001$ \\
\hline Admission (days) & & & $54.7 \pm 18.2$ & 53.5 & $30-134$ & $52.9 \pm 18.8$ & 50.0 & $24-88$ & 0.34 & 0.732 \\
\hline From admission to & & & $11.3 \pm 9.5$ & 8.5 & $0-43$ & $12.7 \pm 12.3$ & 7.0 & $3-52$ & 0.49 & 0.629 \\
\hline From admission to & $n$ of exercis & room (days) & $1.6 \pm 0.7$ & 1.0 & $1-4$ & $1.7 \pm 1.1$ & 1.0 & $1-5$ & 0.77 & 0.443 \\
\hline Item $^{\mathrm{b}}$ & Category & Frequency $(n)$ & & egory & Fre & quency $(n)$ & $U$ & & & $P$ \\
\hline \multirow[t]{6}{*}{ KI } & $\mathrm{B}$ & 1 & $\mathrm{~B}$ & & 0 & & \multicolumn{2}{|c|}{19.50} & & $<0.001$ \\
\hline & $\mathrm{C}$ & 7 & $\mathrm{C}$ & & 0 & & & & & \\
\hline & $\mathrm{D}$ & 6 & $\mathrm{D}$ & & 0 & & & & & \\
\hline & $\mathrm{E}$ & 18 & $\mathrm{E}$ & & 0 & & & & & \\
\hline & $\mathrm{F}$ & 3 & $\mathrm{~F}$ & & 13 & & & & & \\
\hline & $\mathrm{G}$ & 0 & G & & 6 & & & & & \\
\hline \multirow[t]{5}{*}{ JCS } & 0 & 28 & 0 & & 4 & & \multicolumn{2}{|c|}{118.0} & & $<0.001$ \\
\hline & 1 & 5 & 1 & & 6 & & & & & \\
\hline & 2 & 1 & 2 & & 2 & & & & & \\
\hline & 3 & 0 & 3 & & 1 & & & & & \\
\hline & 10 & 1 & 10 & & 6 & & & & & \\
\hline Item $^{\mathrm{c}}$ & Category & Frequency $(n)$ & & egory & Fre & quency $(n)$ & $X^{2}$ & & & $P$ \\
\hline \multirow[t]{2}{*}{ Delirium } & - & 30 & - & & 6 & & \multicolumn{2}{|c|}{16.24} & & $<0.001$ \\
\hline & + & 5 & + & & 13 & & & & & \\
\hline \multirow[t]{3}{*}{ NYHA } & None & 34 & No & & 15 & & \multicolumn{2}{|c|}{ Exact test } & & 0.065 \\
\hline & Slight & 1 & Sli & ght & 2 & & & & & \\
\hline & Severe & 0 & & yere & 2 & & & & & \\
\hline \multirow[t]{2}{*}{ Hugh-Jones } & - & 34 & - & & 18 & & \multirow{2}{*}{\multicolumn{2}{|c|}{ Exact test }} & & 0.584 \\
\hline & + & 1 & + & & 1 & & & & & \\
\hline \multirow[t]{2}{*}{ Brunnstrom Stage } & - & 27 & - & & 16 & & \multirow{2}{*}{\multicolumn{2}{|c|}{ Exact test }} & & 0.405 \\
\hline & + & 8 & + & & 3 & & & & & \\
\hline \multirow[t]{2}{*}{ Orthopedic disease } & - & 33 & - & & 19 & & \multirow{2}{*}{\multicolumn{2}{|c|}{ Exact test }} & & 0.416 \\
\hline & + & 2 & + & & 0 & & & & & \\
\hline
\end{tabular}

${ }^{a}$ MMT, Manual muscle test; HDS-R, Hasegawa's dementia scale; MAT, motor age test; PT, physical therapy

b KI, Katz's index; JCS, Japan coma scale

c NYHA, New York heart Association Classification of cardiac function; Hugh-Jones, Hugh-Jones classification for respiratory disease; Brunnstrom stage, evaluation of paralysis in patients with cerebral stroke 
The walking group included 35 patients, of whom ten used a cane and 25 used a walker. The non-walking group comprised 19 patients, of whom nine required assistance for all movement. Differences between the walking and non-walking groups were noted for all items related to physical and psychological functions and in the NYHA and age (Table 3). Significant correlations were noted among the MMT, MAT, KI and HDS-R scores (Table 4). Discriminant analysis by the stepwise method was applied using KI, HDS-R, MAT, delirium, and NYHA, which were selected as items showing significant differences between the two groups. In MLR analysis using these items, only

Table 4 Pearson's correlation coefficients $(r)$ for items showing significant correlation

\begin{tabular}{lllr}
\hline Items & MAT & KI & HDS-R \\
\hline MMT & 0.59 & -0.42 & 0.43 \\
MAT & & -0.57 & 0.59 \\
KI & & & -0.72 \\
\hline
\end{tabular}

All correlations were significant with $P<0.001$ the MAT and HDS-R were significant. However, based on empirical knowledge, the influence of complications could not be ruled out. Thus, items associated with complications were added one by one to the initial MLR model. Addition of the NYHA caused a significant decrease of $-2 \mathrm{ML}$ (difference: 7.701), an increase in the model $X^{2}$ value (difference: 7.741), and improvement of the discriminant hitting ratio (payoff rate: $3.7 \%$ ). Thus, this model was designated as the final MLR model (MLR model 1: Table 5). Based on the equation shown in Table 5, we calculated a value for $P$. The overall discriminant hitting ratio calculated from the cross table of the measured and predicted values, and the hitting ratios of the walking and non-walking groups were all $94.4 \%$.

Since the weight-bearing exercise was initiated early after surgery, patients may have been unable to utilize their full motor ability. Thus, of the variables selected for the MLR model 1, MAT was replaced with a value measured 1 week later (MAT-1w), and the analysis was repeated. However, the evaluation could not be made after 1 week in four patients because of their poor physical condition and a delay in measurements caused by holiday periods. Thus,

Table 5 Factors affecting gait prognosis: development of a prediction equation

\begin{tabular}{lcllllr}
\hline & $\beta$ & Standard error & $p$ & Odds ratio & \multicolumn{2}{c}{$95 \%$ Confidence interval of odds ratio } \\
\cline { 3 - 6 } & & & & Lower & 1.23 & 1.01 \\
MAT & 1.08 & 0.45 & 0.016 & $2.93^{\mathrm{a}}$ & 1.05 & 24.59 \\
HDS-R & 0.31 & 0.13 & 0.020 & $1.36^{\mathrm{a}}$ & 0.00 & $0.02^{\mathrm{a}}$ \\
NYHA & -4.11 & 3.73 & 0.270 & & 0.00 \\
Const. & -12.9 & 5.01 & 0.010 & & \\
\hline
\end{tabular}

-2 ML $(-2$ maximal likelihood $)=16.71 ;$ Model $X^{2}=53.34(P<0.001)$

Prediction equation: $p=1 /[1+\exp (-1 \times$ Score $)]$

Score $=1.08 \times$ MAT(points) $+0.31 \times$ HDS-R(points $)-4.11 \times$ NYHA $($ category values of none $=0$, mild $=1$, severe $=2)-12.9$

${ }^{a}$ If the odds ratio $>1$, the probability categorized into walking group is higher as the point is larger. If the odds ratio $<1$, the probability categorized into non-walking group is higher as the point is larger

Table 6 Statistical data and prediction equation using MAT evaluated after 1 week (MAT-1w)

\begin{tabular}{|c|c|c|c|c|c|c|}
\hline \multirow[t]{2}{*}{+2} & \multirow[t]{2}{*}{$\beta$} & \multirow[t]{2}{*}{ Standard error } & \multirow[t]{2}{*}{$p$} & \multirow[t]{2}{*}{ Odds ratio } & \multicolumn{2}{|c|}{$95 \%$ Confidence interval of odds ratio } \\
\hline & & & & & Lower & Upper \\
\hline MAT- $1 w^{a}$ & 1.26 & 0.57 & 0.027 & $3.53^{\mathrm{b}}$ & 1.15 & 10.79 \\
\hline HDS-R & 0.43 & 0.23 & 0.063 & $1.53^{\mathrm{b}}$ & 0.98 & 2.40 \\
\hline NYHA & -6.28 & 4.70 & 0.182 & $0.00^{\mathrm{b}}$ & 0.00 & 18.87 \\
\hline Const. & -17.1 & 7.54 & 0.024 & 0.00 & & \\
\hline
\end{tabular}

-2 ML $(-2$ maximal likelihood $)=12.54$; Model $X^{2}=53.87(P<0.001)$

Prediction equation: $p=1 /[1+\exp (-1 \times$ Score $)]$

Score $=1.26 \times$ MAT- $1 \mathrm{w}$ (points $)+0.43 \times$ HDS-R(points $)-6.28 \times$ NYHA(category values of none $=0$, mild $=1$, severe $=2)-17.1$

${ }^{a}$ MAT-1w is motor age test that evaluated after 1 week from initiation of weight-bearing exercise in a rehabilitation room

${ }^{\mathrm{b}}$ If the odds ratio is $>1$, the probability categorized into walking group is higher as the point is larger; if the odds ratio $<1$, the probability categorized into non-walking group is higher as the point is larger 
these patients were excluded from the analysis, and a total of 50 patients (mean age $77.9 \pm 8.4$ years old; walking group, $n=31$; non-walking group, $n=19$ ) were analyzed. The results are shown in Table 6 (MLR model 2). Comparison of $-2 \mathrm{ML}$ showed that the goodness of fit was higher in MLR model 2 than in MLR model 1, and the discriminant hitting ratio of MLR model 2 was $96.0 \%$, with the hitting ratios of the walking and non-walking groups being 96.8 and $94.7 \%$, respectively.

\section{Discussion}

Significant differences were noted in physical and psychological function between the walking and non-walking groups, which is consistent with the results of several earlier studies. Thus, the prediction of gait ability may be possible by evaluating one of these items. However, considering that the determination of gait ability in elderly HF patients is not simple, prediction based on a single item may lead to an incorrect judgment; thus, a combination of two or more items may increase the accuracy of prediction. Hence, construction of a model of prediction from multiple items using a multivariate analytical method may be suitable for predicting gait ability. In support of this, the prediction accuracy of our MLR analysis was high.

Items selected by MLR, such as MAT and HDS-R, were highly correlated with other measures of physical and psychological functions, demonstrating the representative nature of these items. Thus, the influence of both physical and psychological functions needs to be considered for gait prediction. Johnson et al. [12] first described the MAT based on an evaluation table with reference to the typical motor development process between 0 and 72 months (6 years) after birth. However, this test is now frequently used in the field of rehabilitation. The decline of motor function with age is markedly influenced by neurology. Such a decline does not necessarily regress to the childhood development process, but it may be represented based on standard and objective indices using the MAT, and an overall evaluation including balance ability and muscle strength may also be possible. In addition, no specific instrument is needed for the evaluation, and the procedure is simple. The HDS- $\mathrm{R}$ is a method of evaluating intelligence that was designed in Japan, and its usefulness has also been confirmed in other countries [14].

Although the NYHA was not significant in the MLR, we added it to the prediction equation because it has been reported to influence prognosis $[6,7]$. Since the NYHA score was not significant in the initial model, its inclusion was not strictly necessary, but it may become significant in a patient population comprising an increased number of subjects with the complication of heart disease; the number of patients with heart disease in the current study was relatively small.

The patients may not have been able to use their full motor ability on the day of initiation of weight-bearing exercise because they were elderly and post-surgery interval was short. Hence, MAT in MLR model 1 was replaced with MAT-1w. In MLR model 2, the OR of MAT-1w was improved, and the accuracy of prediction increased. It is understandable that the use of the value after the 1-week period increased the accuracy of prediction, but MLR model 1 was also significant and had a high hitting ratio. The precision of the prediction was quite high with both models. Therefore, MLR model 1 may be fully applicable in terms of early prediction of gait ability, making these findings useful for planning a PT strategy. Since the model is capable of predicting the appropriate target strategy at an early stage, assessments of the requirement for continuation of PT and the utilization of social resources upon early discharge may possibly be made more easily.

Prolongation of the injury-associated recumbency period (the period between admission and PT) increases the possibility of abasia, but there was no significant difference in the duration of this period between the groups, and no patients were recumbent for a prolonged period after injury. Moreover, the patients for whom PT was suspended for a prolonged period were excluded from the study. Thus, construction of multiple models that take these conditions into account will be necessary to further extend the work presented here. In addition, changes due to differences in rehabilitation or the ward system among facilities and developments in surgical therapy will also need to be accounted for in future models. Nonetheless, the model that we have constructed should prove useful for prediction of gait ability in hip-fracture patients. Accumulation of data regarding the items selected for the prediction equation at each institution and application of the model using these data may lead to the construction of a model with even higher accuracy.

\section{References}

1. Bitsch M, Foss N, Kristensen B, Kehlet H. Pathogenesis of and management strategies for postoperative delirium after hip fracture: a review. Acta Orthop Scand. 2004;75:378-89.

2. Tanaka J, Tokimura F, Seki N. Outcomes of hip fracture surgery in patients aged $\geq 90$ years. Orthopedics. 2003;26:55-8.

3. Marcantonio ER, Flacker JM, Michaels M, Resnick NM. Delirium is independently associated with poor functional recovery after hip fracture. J Am Geriatr Soc. 2000;48:618-24.

4. Duppils GS, Wikblad K. Delirium: behavioural changes before and during the prodromal phase. J Clin Nurs. 2004;13:609-16.

5. Binder EF, Brown M, Sinacore DR, Steger-May K, Yarasheski KE, Schechtman KB. Effects of extended outpatient rehabilitation 
after hip fracture: a randomized controlled trial. JAMA. 2004;292:837-46.

6. Masuda T, Miura N, Ishii S, Hibino Y, Beppu M. New preoperative evaluation system of the physical findings of aged patients with femoral neck fracture. J Orthop Sci. 2004;9:434-9.

7. Zanocchi M, Maero B, Francisetti F, Giona E, Nicola E, Margolicci A, et al. Multidimensional assessment and risk factors for prolonged hospitalization in the elderly. Aging Clin Exp Res. 2003;15:305-9.

8. Choong PF, Langford AK, Dowsey MM, Santamaria NM. Clinical pathway for fractured neck of femur: a prospective, controlled study. Med J Aust. 2000;172:423-6.

9. Jarnlo GB, Ceder L, Thorngren KG. Early rehabilitation at home of elderly patients with hip fractures and consumption of resources in primary care. Scand J Prim Health Care. 1984; 2:105-12.

10. Crotty M, Whitehead CH, Gray S, Finucane PM. Early discharge and home rehabilitation after hip fracture achieves functional improvements: a randomized controlled trial. Clin Rehabil. 2002;16:406-13.

11. Lucille D, Catherine W. Muscle testing, 5th edn. Philadelphia: WB Saunders; 1986.
12. Johnson MK, Zukc FN, Wingate K. The motor age test. J Bone Joint Surg. 1951;33-A:698-707.

13. Katz S, Ford AB, Moskowitz RW, Jackson BA, Jaffe MW. Studies of illness in the aged: the index of ADL, a standardized measure of biological and psychosocial function. JAMA. 1963;185:914-9.

14. Kim KW, Lee DY, Jhoo JH, Youn JC, Suh YJ, Jun YH, et al. Diagnostic accuracy of mini-mental status examination and revised Hasegawa dementia scale for Alzheimer's disease. Dement Geriatr Cogn Disord. 2005;19:324-30.

15. Committee on Medical Rating of Physical Impairment. Guides to the evaluation of permanent impairment; the cardiovascular system. JAMA. 1960;172:1049-60.

16. Hugh-Jones S. A simple standard exercise test and its use for measuring exertion distress. Br Med J. 1952;4749:65-71.

17. Brunnstrom S. Movement therapy in hemiplegia. New York: Harper \& Row; 1970.

18. David WH, Stanley L. Applied logistic regression. New York: John Wiley \& Sons; 1989.

19. Daniels L, Worthingham C. Muscle testing: technqiues of manual examination. Philadelphia: W.B. Saunders; 1980. 